\title{
3D Imaging of Biological Cells Using a CryoFIB/SEM and a CryoTEM
}

\author{
Korrinn Strunk*, Trevor Clark**, Jennifer L. Gray*, Peijun Zhang*** \\ *Department of Mechanical Engineering and Materials Science, University of Pittsburgh, \\ Pittsburgh, PA \\ **Materials Research Institute, Pennsylvania State University, University Park, PA \\ ***Department of Structural Biology, University of Pittsburgh School of Medicine, Pittsburgh, PA
}

The emerging discipline of cryo-electron tomography provides unique opportunities to determine 3dimensional (3D) cellular architectures in their native conditions. However, one major limitation, specimen thickness, has hindered its broader application in cellular structural biology, since it's performed in TEM by tilting the specimen through a series of angles. Thinning a biological sample using cryo-ultramicrotomy and a diamond knife has yielded limited success due to technical difficulties and artifacts associated with this mechanical sectioning method [1]. It has been shown that a dual beam system consisting of a scanning electron microscope (SEM) and focused ion beam (FIB) used under cryogenic conditions can successfully mill biological samples for use in cryoTEM. This technique eliminates the cutting artifacts that are associated with ultramicrotomy, while thinning specimens enough for electron tomography $[2,3]$.

We explored the possibility of using a cryo-FIB dual beam system to thin portions of cultured HeLa cells using very low incident beam angles and currents of about $10 \mathrm{pA}$. The Hela cells were grown on gold grids that were plunge frozen and then milled using an FEI Quanta 3D dual beam system and imaged using an FEI Polara TEM. The milling angles were generally kept between $10^{\circ}$ and $20^{\circ}$ depending on the specimen area. A schematic of the milling conditions is shown in figure 1a and the milled sample in the FIB is shown in figure 1b. Figure 2 shows the milled area imaged in the TEM. It can be seen that the ice remains vitreous throughout the entire process. Large protein complexes are clearly visible and appear intact, along with vesicles and cellular filaments deep inside of the HeLa cell. Cryo-electron tomographic analysis of such regions will further allow us to explore the internal 3D architectures of native mammalian cells.

References:

[1] A. Al-Amoudi, et al., Journal of Structural Biology, vol. 150, no. 1, pp. 109-121, Apr. 2005

[2] M. Marko, et al., Journal of Microscopy, vol. 222, no. 1, pp. 42-47, 2006

[3] A. Rigort et al., Journal of Structural Biology, pp. 160-79 2010

[4] This work was supported by NIH grant R21RR024424. The authors would also like to thank Travis Wheeler and the University of Pittsburgh School of Medicine Machine Shop 
a)

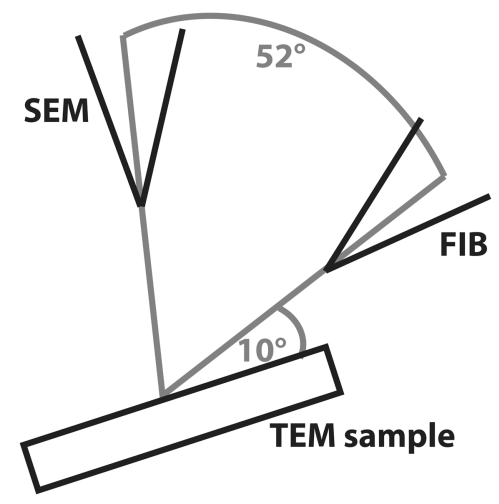

b)

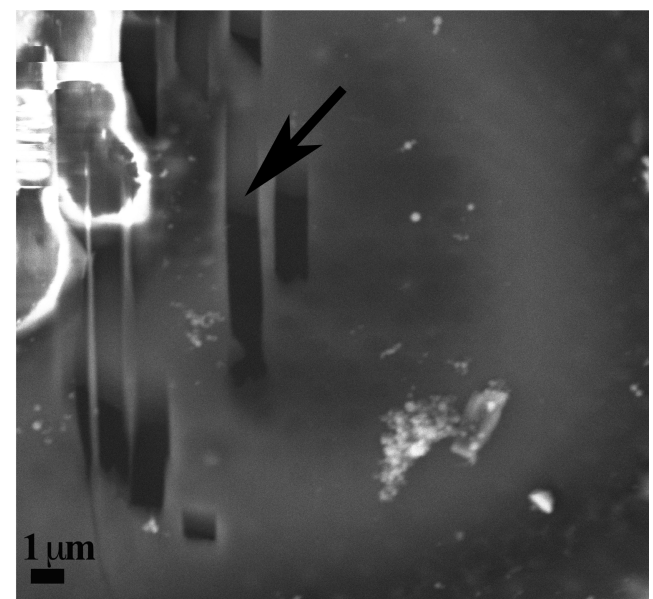

Figure 1: a) A schematic drawing of the set up of the dual beam FIB/SEM system. b) The milled sections imaged using the SEM in the dual beam. The arrow indicates the section imaged in figure 2.
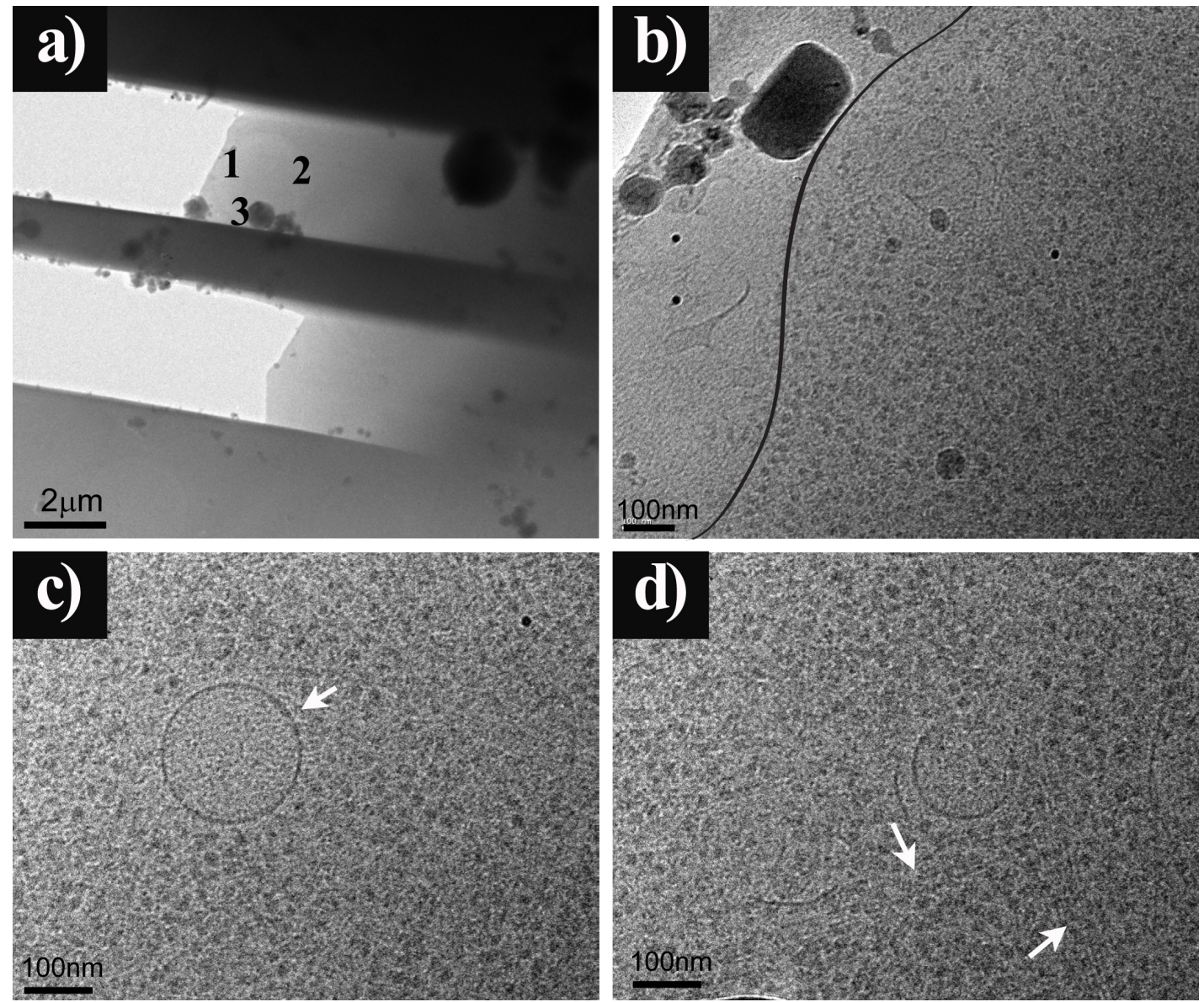

Figure 2: a) The milled sample imaged in the cryo-TEM. The parts of the image labeled 1, 2 and 3 correspond to the magnified images in panels b), c) and d) respectively. Large protein complexes are abundant inside of the HaLa cell, along with vesicles, microtubules and cellular filaments (arrowhead). The cell boundary is outlined in panel $\mathrm{b}$ for clarity. The dark particles are $15 \mathrm{~nm}$ gold beads applied to the sample when plunge freezing. 